\title{
"Dessine-moi tes langues et je te dirai qui tu es" : le rapport des enfants lusodescendants au portugais comme Langue-Culture d'Origine en Allemagne
}

\section{Sílvia Melo-Pfeifer et Alexandra Schmidt}

\section{(2) OpenEdition Journals}

Édition électronique

URL : http://journals.openedition.org/rdlc/1512

DOI : $10.4000 /$ rdlc. 1512

ISSN : 1958-5772

Éditeur

ACEDLE

Référence électronique

Sílvia Melo-Pfeifer et Alexandra Schmidt, «"Dessine-moi tes langues et je te dirai qui tu es" : le rapport des enfants lusodescendants au portugais comme Langue-Culture d'Origine en Allemagne ",

Recherches en didactique des langues et des cultures [En ligne], 10-1 | 2013, mis en ligne le 07 janvier 2013, consulté le 19 avril 2019. URL : http://journals.openedition.org/rdlc/1512 ; DOI : 10.4000/ rdlc. 1512

Ce document a été généré automatiquement le 19 avril 2019

\section{(c) (†) $\odot$}

Recherches en didactique des langues et des cultures is licensed under a Creative Commons AttributionNonCommercial-NoDerivatives 4.0 International License 


\title{
"Dessine-moi tes langues et je te dirai qui tu es" : le rapport des enfants lusodescendants au portugais comme Langue-Culture d'Origine en Allemagne
}

\author{
Sílvia Melo-Pfeifer et Alexandra Schmidt
}

\section{NOTE DE L'ÉDITEUR}

Cette étude a été réalisée dans le cadre du projet "Imagens do (Ensino) Português no Estrangeiro" (2011-2013), sous la coordination de S. Melo-Pfeifer \& A. Schmidt et avec le soutien de Camões, IP et de l'Ambassade de Portugal à Berlin. Pour plus d'informations : http://cepealemanha.wordpress.com/imagens-do-ensino-portugues-no-estrangeiro/.

\section{Introduction}

L'enseignement des langues-culture d'origine (Elco), ainsi que les concepts et les pratiques qui le fondent, connaît à présent un nouvel essor réflexif en conséquence des récents développements théoriques autour des concepts "Heritage Language Education" (Brinton et al, 2007 ; Carreira \& Kagan, 2011) et Compétence Plurilingue (Little, 2010). Les pratiques Elco restent, malgré toutes les controverses et limitations conjoncturelles (Castellotti, 2010; Dabène, 1989), bien ancrées dans les modèles d'enseignementapprentissage esquissés il y a déjà quelques décennies (Bertucci \& Corblin, 2007 ; Dabène, 1989), basés sur les supposés (et homogènes!) besoins linguistiques, communicatifs, pragmatiques et identitaires des enfants issus de l'immigration, en vue soit de leur intégration dans le pays d'accueil, soit de leur retour au pays d'origine (Castellotti, 2010). 
2 Nous croyons, en effet, que, grâce aux théories et aux champs d'études émergents, il faut à présent s'engager dans deux discussions très pressantes : d'un côté, la distinction entre les concepts "LM", "LE", "L2" et "Heritage Language" ou "Langue Culture d'Origine", pour en percevoir les rapprochements, les frontières, les espaces de contact et/ou de traverse ainsi que leurs limitations heuristiques ; d'un autre côté, l'insertion de ces discussions sur les Elco dans un espace didactologique réflexif plus souple, pour montrer comment cette conception peut s'enrichir à travers le dialogue avec les approches plurielles de l'enseignement-apprentissage des langues (notamment l'Intercompréhension, la Didactique Intégrée et l'Enseignement d'une Matière Intégrée à une Langue - Candelier $e t$ al, 2007) dans le cadre du développement de la Compétence Plurilingue et Interculturelle des enfants migrants (Castellotti \& Moore, 2010 ; Fürstenau \& Gomolla, 2011).

Dans le cadre de ces problématiques, nous chercherons, dans cette contribution, à comprendre le rapport des publics de cet enseignement à la Langue-Culture d'Origine (LCO), afin de saisir comment cet enseignement enrichit le répertoire plurilingue des sujets et contribue à la construction d'une identité plurielle. Pour ce faire, nous avons recueilli, à l'aide de 34 enseignants de Portugais Langue-Culture d'Origine (Plco), 984 dessins auprès des enfants issus de l'immigration de l'espace lusophone, habitant à présent en Allemagne (Melo-Pfeifer \& Schmidt, 2012a). S'agissant d'un public âgé de 6 à 12 ans, cette méthodologie comporte plusieurs avantages pour saisir le rapport des enfants aux langues, surtout quand ils sont encore en début de scolarisation (Molinié, 2010 ; Perregaux, 2011), puisqu'elle permet d'appréhender la richesse des représentations sans (trop) faire appel au langage écrit.

Notre contribution sera développée en trois temps :

- réflexion théorique concernant le concept d'Elco et son rapport à d'autres concepts tels que LM, LE, L2 et "Heritage Language", ainsi que son rôle actuel dans le cadre d'une Didactique des Langues et du Plurilinguisme ;

- analyse empirique du corpus de dessins recueillis auprès des enfants, en termes de représentations concernant le Plco et le rôle de cette langue dans les dynamiques linguistiques et identitaires en cours chez eux ;

- articulation de la réflexion théorique avec les images/représentations recueillies à propos du portugais, afin de dégager les spécificités de ce "profil d'apprenant" et des perspectives de développement des Elco, notamment en ce qui concerne l'articulation avec des approches plurielles des langues-cultures.

\section{Compétence plurilingue et répertoires pluriels : rôles et places pour la LCO?}

5 Le développement de la compétence plurilingue (dans ses différentes composantes affectives, cognitives, linguistiques...) prend les répertoires dynamiques et composites des sujets en tant que réalités-phares dans un monde hypercomplexe. La place des compétences linguistiques dans ce contexte, calquée d'une vision essentialiste et étanche des langues encore fortement ancrée dans le sujet natif, est mise en cause par le besoin même de développement de ces répertoires pluriels et flexibles, dynamiques et déployés en situation.

6 La notion de compétence plurilingue valorise l'éventail de ressources linguistiques, socioaffectives, communicatives et cognitives des sujets, afin de bâtir une compétence (à la fois 
plurilingue et interculturelle) solide et intégrée, une capacité à "translanguaging" (García, 2009) dans plusieurs contextes, à l'aide de tout le capital de ressources disponibles et en construction. Le but est la construction, la mobilisation et l'intégration consciente de répertoires linguistiques variés et partiels, qui seraient disponibles à des fins concrètes, dans de situations tangibles :

There is nothing wrong with that phenomenon of partial competence: no one needs all the resources that a language potentially provides (...). Our real "language" is very much a biographical given, the structure of which reflects our own histories and those of the communities in which we spend our lives. (Blommaert, 2010: 103)

Le développement situé des ressources linguistiques implique, en outre, l'articulation avec divers indices de contextualisation et avec une multitude de ressources sémiotiques. Ceci dit, le développement de la compétence plurilingue présuppose une approche multimodale des situations de communication où les langues ne sont que l'un des ingrédients.

La notion de Compétence Plurilingue considère les soi-disant répertoires sémiotiques des sujets comme des "mobile resources" (Blommaert, 2010), développées, adaptées, (ré/ dé)valorisées et investies ou désinvesties par rapport aux contextes sociaux, aux situations de communication et d'action et aux besoins des sujets. Dans ce sens, par rapport à la notion de "répertoire", qui induit une compréhension plutôt statique/ descriptive des savoirs et des savoir-faire des sujets, celle de "ressources" implique un usage en contexte et un développement au cours de l'interaction sociale, par rapport à la multiplicité de paramètres qui la caractérise. Selon cet auteur encore, cet ensemble de ressources mobiles et plurilingues est le fruit des mobilités diverses (que ce soit géographiques ou virtuelles) que le sujet subit tout au long de sa vie, au contact avec plusieurs interlocuteurs et avec différentes sources et ressources discursives et linguistiques. Dans ce cadre, la biographie (linguistique) des sujets devient un facteur central pour comprendre le "puzzle" des ressources linguistiques et sémiotiques des sujets (les ressources linguistiques intégrant ceux sémiotiques), c'est-à-dire, leur répertoire plurilingue et sa mise en action. De la même façon, et si l'étalon n'est plus celui du sujet natif mystifié, mais bien celui des besoins du locuteur réel (que ce soit par rapport à une supposée langue maternelle ou étrangère), alors la Compétence Plurilingue rend compte de parcours collaboratifs d'élargissement des répertoires des sujets, non plus dans une tradition épistémologique cognitiviste (où un apprenant donné développe ses savoirs et savoirs-faire), mais plutôt d'un point de vue socioconstructiviste, où les sujets agissent ensemble avec et par leurs ressources linguistiques et sémiotiques, qu'ils partagent, mutualisent et développent ensemble (Bono \& Melo-Pfeifer, 2012).

Or, l'une de ces ressources qui compose le répertoire plurilingue des sujets, surtout en contextes de mobilité tels que l'immigration, c'est la langue d'origine (ou "heritage language", comme nous avons tendance à privilégier, Melo-Pfeifer \& Schmidt, 2012a). Même désignant cette langue comme ressource dans le cadre d'un répertoire plus complexe, nous savons que les connaissances dans cette langue d'origine sont, en effet, un ensemble complexe et partiel de ressources à la fois linguistiques, pragmatiques, idéologiques. Ce sont, en effet, des ressources "tronquées", pour reprendre Blommaert, puisque développées à des fins diverses, dans des situations dynamiques induisant des dynamiques linguistiques et identitaires :

Immigrant children, for instance, may grow up in a family in which they hear their parents speak a language. They can understand this language, and respond adequately to utterances made in it, but they never learn to speak it. This does not 
mean that this language does not belong to the children's repertoire: it does belong

to it, be it only in a minimal and receptive form. (Blommaert, 2010: 106).

10 La langue d'origine est donc, à notre avis, un exemple assez clair pour comprendre la variabilité et le déséquilibre de la compétence plurilingue des sujets (Coste, Moore \& Zarate, 2009), d'un côté, et la pertinence des approches plurielles dans le développement de cette compétence, de l'autre côté. Ainsi, même si cette langue ne fait pas souvent objet d'un enseignement formel, elle constitue néanmoins une ressource individuelle qui peut être investie dans le contact avec plusieurs langues et cultures, en tant que tremplin cognitif et affectif soit vers la(les) langue(s) du pays d'accueil, soit vers la compréhension de la diversité, la sienne et celle des autres.

11 La langue d'origine, en tant que "ressource biographique", est donc l'un des constituants de la compétence plurilingue individuelle, et ainsi, un atout dans un monde de mobilité, de besoins linguistiques et communicatifs croissants et d'accélération des échanges. Elle est aussi une occasion de rapprochement entre les sujets et de construction de situations de travail et de communication collaboratives.

\section{Langue-Culture d'Origine et "Heritage Language" : des concepts au carrefour de la LM, la LE et la L2}

12 La Didactique des Langues (désormais DL) se voit souvent confrontée à des concepts qui entraînent entre eux des rapports assez proches ou nuancés (pour le cas de concepts souvent pris comme synonymes, hyperonymes et hyponymes, voir Melo, 2006). Dans d'autres cas, c'est l'évolution même des contextes sociaux et des approches épistémologiques qui interroge la capacité des concepts à expliquer et à décrire les réalités sociales (Bono \& Melo-Pfeifer, 2012; Dabène, 2000) et donc la légitimité heuristique des "dénominations reçues" (Cuq, $2003: 151)$.

Pour ce qui est des concepts qui mobilisent le "rapport biographique" des sujets aux langues (ordre d'acquisition, statut social et officiel des langues...), plusieurs sont les fluctuations terminologiques, du fait même des contextes hypercomplexes où les langues sont apprises, mobilisées, (re)utilisées, ignorées et oubliées. Autrement dit, les dynamiques associées à la mobilité croissante (en termes du statut des sujets et de leurs langues et aussi des variations familiales, culturelles, économiques et politiques complexes) rendent nécessaire d'expliciter le regard que l'on porte sur les concepts que l'on utilise pour les décrire et analyser.

De son côté, la notion de LM "renvoie à la combinaison de deux séries de deux facteurs au moins: l'ordre de l'acquisition et l'ordre du contexte. Il s'agirait de dénommer ainsi la langue acquise la première par le sujet parlant dans un contexte où elle est aussi la langue utilisée au sein de la communication. Le caractère spontané, naturel de son usage, l'aisance de son maniement, apparaissent parfois comme des traits définitoires de la langue maternelle" (Cuq, 2003 : 151). Ces traits définitoires, que l'auteur conteste ouvertement, ne rendent pas compte de "la complexification du statut des langues et de leur rôle chez les sujets parlants" (idem), ainsi que des changements plus au moins subis tout au long de la construction de la biographie linguistique.

Dans ce sens, le concept de LM ne peut pas toujours être opposé à celui de Langue Étrangère, associé souvent à des contextes d'apprentissage formels, avec un moindre degré d'attachement socio-affectif et d'appartenance. En conséquence, l'ouverture 
théorique et heuristique des concepts à des postures hybrides et la reconnaissance de statuts mixtes, intermédiaires et transitoires, nous amènent à revisiter d'autres concepts, notamment ceux de L2 et de Langue d'Origine. Ainsi, comme l'explicite Moussouri,

Les notions de langue seconde et de langue d'origine se définissent par des facteurs d'ordre sociolinguistique, à la fois objectifs et subjectifs. Les facteurs sociolinguistiques objectifs concernent le positionnement des langues dans des situations sociales et linguistiques définies par des politiques linguistiques. Les facteurs sociolinguistiques subjectifs portent sur les représentations que se font les locuteurs des langues en présence sur ces mêmes langues. (2010:143).

Ainsi, la L2 est censée être l'une des langues officielles dans un paysage linguistique donné, avec un statut reconnu et avec une circulation publique. Cependant, elle n'est souvent ni la "première" d'une partie plus ou moins importante de la population, ni une langue de scolarisation. Dans d'autres acceptions, la L2 est la langue enseignée aux adultes en situation de migration, à travers des dispositifs spécifiques de formation, c'està-dire, la langue du pays d'accueil. Ce serait, dans cette acception, la langue des migrants de la première génération. Une troisième acception sera celle qui lie L2 à un certain ordre chronologique dans l'apprentissage.

La notion LCO renvoie, de son côté, aux langues avec lesquelles les sujets sont censés être en contact au sein de la famille et/ou de la communauté migrante dans un certain pays d'accueil. C'est donc une "langue d'ailleurs", qui rapporte d'une "perspective à la fois monocentrée et trajectoriale" (Castellotti, 2010: 89), en opposition à la/aux langue/s nationales. Cependant, il faut rappeler que :

- d'un point de vue sociolinguistique, la langue d'origine peut ne pas être attachée à des mouvements migratoires, à cause des multiples contextes de mobilité, plus ou moins individuels; ce concept peut encore se décliner au pluriel, dans le cas des familles bilingues dans un troisième environnement linguistique (par exemple, un enfant d'un couple italienalbanais, parlant les deux langues à la maison, habitant en Allemagne); finalement, il s'agit d'une notion souvent sans référent réel, dans le cas où cette langue a été abandonnée au cours des parcours de vie et des générations, au profit d'autres langues (on parle, dans ces cas, de "language attrition" (voir les travaux présents dans Cook, 2003);

- d'un point de vue socio-affectif, la Langue d'Origine peut être, à la fois, i) cachée par les sujets, qui ne veulent pas la reconnaître ou se reconnaître comme appartenant à une couche sociolinguistique donnée, et ii) assignée par la société "d'accueil" (surtout quand on ne se sent pas accueilli !); sa valeur affective est donc souvent questionnable et problématique ; comme le remarque V. Castellotti, "catégoriser les enfants à partir de leur origine supposée, reconstruite, fantasmée, conduit à déterminer pour eux ces origines, à les fossiliser, à les leur assigner" (2010: 89);

- d'un point de vue scolaire, la Langue d'Origine peut être une des langues de scolarisation (dans le cas des sections européennes ou des écoles bilingues) et/ou un objet extra-scolaire en dehors du curriculum (dans un système d'enseignement appelé "parallèle"); en même temps, elle peut être encore apprise à l'école en tant que langue étrangère, dans le cas où le système scolaire lui alloue le statut d'option linguistique ;

- du point de vue de son acquisition et utilisation, la Langue d'Origine peut avoir été apprise à la maison, avant la scolarisation (cas où l'on approche souvent de la désignée Langue Maternelle), ou ne pas avoir été apprise qu'à l'école (cas où on se rapproche des propriétés assignées à une Langue Étrangère); dans le premier cas, il s'agit d'une langue parlée et utilisée couramment à la maison (faisant partie des "rituels de littératie" au sein de la famille), tandis que, dans le second cas, il s'agit d'une situation où, bien qu'ayant encore un 
statut spécial, elle ne sert pas (ou plus) comme langue (principale) de communication; le soutien et la reconnaissance de la communauté (d'origine et d'accueil) semblent être importants pour le maintien de la langue d'origine au sein de la famille.

\section{concept de LCO met en évidence le caractère hybride, mixte et transitoire des ressources} linguistiques dont nous avons parlé auparavant :

It thus refers to a language with an "in-between" status in terms of mother tongue/ foreign language and formal/informal instruction, depending on the role it plays in speakers' daily life and on the relationship they entail with it (...). In terms of Language Education, Heritage Language pupils' and students' skills are quite heterogeneous and asymmetrical - with highly developed listening comprehension and interaction skills - in what we could call a continuum between productive and receptive skills (Melo-Pfeifer \& Schmidt, 2012a: 3).

\section{Le projet "Images (de l'enseignement) du Portugais à l'Étranger" : objectifs et démarches méthodologiques}

19 Le projet "Images (de l'enseignement) du Portugais à l'Étranger" prend le concept d'image au centre de l'approche méthodologique (design du projet et moyens de diagnostic) et de la réflexion en termes d'aboutissements pratiques (impact des images dans l'enseignement-apprentissage et création de parcours de formation pour les professeurs de portugais à l'étranger - ici, hors Portugal).

\section{Un concept central}

Les représentations sociales, en tant que "produit d'un travail social collectif, à travers lequel les agents sociaux construisent leurs modes de connaissance de la réalité" (Zarate, 1993 : 29), constituent des objets d'expression des acteurs sociaux et jouent trois fonctions principales: explication et orientation des comportements individuels et des rapports sociaux, différentiation des groupes et co-construction de la communication, puisque constituant un système de catégorisation et d'interprétation collectif et partagé. Dans ce sens, la représentation sociale est comprise en tant que "passerelle entre le monde individuel et le monde social" (Moscovici, 1989: 99) ou bien, selon Py, une "microthéorie 'économique' en ce sens qu'elle réunit en elle une grande simplicité et un vaste domaine d'application" (2004:8).

En ce qui concerne la DL, le concept de représentation, souvent pris comme synonyme de celui d'image (comme nous le ferons aussi) et très proche de celui de stéréotype (Boyer, 1997), surgit comme mot-clé d'études qui essayent de cerner son influence dans la communication interculturelle et dans l'enseignement-apprentissage des langues (Araújo e Sá et al, 2012). Il est donc considéré comme un concept qui rend compte des a priori qui constituent l'interaction, car il s'assume en tant qu' "outil nécessaire à l'anticipation des conduites et des rôles avant la rencontre" (Yanaprasart, 2002 : 60). Dans ces études en DL, le concept de représentation rend compte des rapports que les apprenants établissent avec les langues qu'ils étudient, d'un côté, et avec les offres curriculaires, de l'autre côté (cf. Candelier \& Hermann-Brennecke, 1993 ; Castellotti \& Moore, 2002). En plus, un grand nombre d'études témoigne de la diversité des fonctions que les représentations assument tout au long de l'interaction, qu'elle soit ou non interculturelle et plurilingue : moteur et 
support des échanges intra- et exo-groupes, outils cognitifs et sémiotiques, marqueurs d'identité, signes de cohésion et de bonheur conversationnel (De Pietro \& Müller, 1997; Deprez, 1997 ; Melo, 2006).

Ces études prouvent que les images sont fortement ancrées dans des processus cognitifs, discursifs, socio-historiques et identitaires, propres à chaque groupe et à chaque sujet. Dans ce sens, les représentations font partie de la façon dont chaque groupe et chaque individu construit et apréhende la réalité (Araújo e Sá \& Pinto, 2006). Plusieurs facteurs sont à la genèse des représentations sociales, notamment de nature identitaire, socio-économique, sociolinguistique, culturelle, politiques et éducatives, induisant, dans le cadre de la DL, une hiérarchisation des langues et des compétences en langues, que ce soit dans le marché du travail, dans les pratiques scolaires, dans les offres curriculaires ou encore dans les projets linguistiques des individus et des groupes sociaux (Araújo e Sá \& Pinho, 2006 ; Candelier, 1997 ; De Pietro, 1995 ; Deprez, 1997 ; Zarate, 1995 et 1997).

Parce que les images sont des objets contextualisés et donc mutables, les conclusions des études qui se penchent sur ce concept sont elles aussi contextualisées et possèdent une validité temporelle et géographique (par exemple, Araújo e Sá \& Schmidt, 2008 ; MeloPfeifer \& Pinto, 2009 ; Pinto, 2005 ; Schmidt, 2011, en contexte portugais). Les images sont, simultanément, des a priori communicatifs et interprétatifs et des objets communicatifs, pouvant être mobilisés et renégociés tout au long de l'interaction (Melo, 2006) ; ainsi, les méthodes de diagnostic de ces images suivent deux tendances principales: la première cerne les images en tant que pré-construction, dans son contenu déclaratif et plus au moins décontextualisé, c'est à dire, "comme une valeur absolue qui permet d'expliquer un certain nombre de comportements langagiers propres à un groupe ou a une collectivité" (Gajo, 2000: 9; aussi Py, 2004); la deuxième analyse l'émergence et la négociation des images au long de l'interaction, afin d'en dégager les éléments thématisés et rendus saillants et les processus ainsi que les produits de la négociation (Melo, 2006). La première de ces approches peut être désignée de "plus objectivante" et la deuxième de "moins objectivante" ou "socioconstructiviste" (Araújo e Sá \& Pinto, 2006, pour une synthèse).

Notre approche, au long du projet "Images (de l'enseignement) du Portugais à l'Étranger" est de nature "plus objectivante" puisque nous cherchons à cerner les représentations de différents publics concernant le portugais (langue maternelle, langue étrangère et langue d'origine) hors frontières portugaises. L'objectif, comme nous le verrons par la suite, c'est la rentabilisation de ce travail de diagnostic dans la construction de parcours différenciés de formation de formateurs pour travailler dans les différents contextes.

\section{Objectifs et phases du projet}

Le projet "Images (de l'enseignement) du Portugais à l'Étranger" poursuit trois objectifs principaux, ceux-ci autour de trois axes: le diagnostic, la compréhension et la rentabilisation des représentations dans différents contextes d'enseignementapprentissage du portugais. Ce projet vise à :

- cerner les représentations de différents acteurs et publics par rapport au Plco (maîtres, enseignants, lecteurs de portugais, parents et apprenants, membres des communautés portugaises à l'étranger) dans différents contextes d'enseignement (intégré, "parallèle", cours livres et optionnels...);

- comprendre les éléments constants et les variations des représentations concernant le PLE, le PLM et le Plco auprès des différents publics (selon leurs propres classifications); 
- créer des parcours de formation de formateurs ancrés dans les représentations plus saillantes, orientés vers la reconstruction ou déconstruction des images du portugais. cerner les représentations, nous avons mis au point deux méthodologies de recueil de données :

- un questionnaire "en ligne" destiné à tous les publics (sauf les enfants), avec une section différenciée (de caractérisation sociolinguistique des différents publics) et une partie commune (de diagnostic des représentations), afin de permettre la comparabilité des résultats (Melo-Pfeifer \& Schmidt, 2012b, pour une analyse de ces questionnaires);

- le recueil de dessins des enfants (entre 6 et 12 ans) participant aux cours de Plco en Allemagne et au Royaume-Uni (ceux-ci restant pour le moment inexploités).

Pour ce qui est de l'objectif numéro deux, celui de comprendre les aspects communs et les différences des représentations auprès de différents publics et contextes, nous croiserons les réponses obtenues à travers la partie commune et la partie ciblée du questionnaire "en ligne": ceci permettra de concilier une approche quantitative préalable et une approche plus qualitative et compréhensive des phénomènes observés. La question centrale est de savoir ce qui décrit le rapport des différents publics au PLM, au PLE et au Plco les différences et les interceptions. Les deux hypothèses sous-jacentes à cette comparaison sont les suivantes :

- les rapports biographiques avec le portugais influencent le contenu des images de cette langue construites au long de la vie ;

- ces images induisent différentes stratégies et dynamiques d'action en contexte éducatif.

En ce qui concerne le troisième objectif - la création de parcours de formation - un prototype de formation est en train d'être créé. Il sera premièrement testé en présentiel auprès d'un public plus restreint et, après une évaluation et adaptation de la formation, il sera transformé dans une formation e-learning, ciblant un public plus vaste: tous les formateurs impliqués dans l'enseignement du portugais à l'étranger.

\section{L'étude empirique : analyse du corpus de dessins recueillis auprès des enfants}

\section{Participants et corpus}

Nous avons recueilli 956 dessins auprès d'enfants prenant des cours de Plco en Allemagne, âgés d'entre 6 et 12 ans, dans plusieurs états fédéraux :

Tableau 1. Corpus recueilli

\begin{tabular}{|l|l|}
\hline État Fédéral & Dessins recueillis \\
\hline Bade-Wurtemberg & 583 \\
\hline Berlin & 14 \\
\hline Brême & 22 \\
\hline
\end{tabular}




\begin{tabular}{|l|l|}
\hline Hambourg & 143 \\
\hline Hesse & 43 \\
\hline Basse-Saxe & 54 \\
\hline Rhénanie-du-Nord-Westphalie & 97 \\
\hline Total & 953 \\
\hline
\end{tabular}

Pour recueillir les dessins auprès des enfants, nous avons pu compter avec l'aide de 34 professeurs travaillant avec différentes communautés portugaises en Allemagne (à travers Camões, $\mathrm{IP}^{1}$ ). Cela signifie que, outre le caractère familier de la tâche pour ce public, la tâche elle-même a été développée pendant les cours, ce qui lui a donné un caractère plutôt scolaire mais qui, en même temps, a permis de valoriser les répertoires dessinés et les expériences vécues hors-école, mais remémorées et mises en évidence.

La consigne donnée était "dessine-toi en train de parler les langues que tu connais". Cette consigne aspirait :

- à mettre l'enfant plurilingue au centre de la tâche réflexive, en inspirant une posture valorisant le vécu (Molinié, 2009 ; Moore \& Castellotti, 2011 ; Perregaux, 2011);

- à mettre en évidence les répertoires pluriels des enfants ainsi que leurs rapports, sans les hiérarchiser en termes de lieu d'apprentissage (à l'école ou ailleurs), d'auto-évaluation de compétences (langues mieux maîtrisées...) ou d'attachement affectif (langues mal ou bienaimées).

Malgré cette aspiration à la clarté et à l'objectivité, besoin est de dire que les enfants ont tout de suite adopté différentes postures par rapport à la consigne, ce qui fait l'originalité et la diversité des dessins recueillis : il y a des enfants qui prennent la consigne plus au pied de la lettre que d'autres. Quelques-uns se dessinent exclusivement eux-mêmes en train de parler (l'activité étant représentée par des bulles de dialogue), comme un portrait plurilingue; d'autres en train d'apprendre une langue à l'école (avec tous les outils qui constituent l'imaginaire de l'apprentissage scolaire des langues) et d'autres en situations communicatives de contact avec d'autres langues dans un cadre social; d'autres encore, finalement, dessinent leur entourage multilingue et les signes culturels qu'ils y attachent.

Il faut encore ajouter que, bien que la tâche ne l'inspire pas, les hiérarchies entre les langues apparaissent plus au moins mises en évidence : les langues de l'école ou apprises à l'école, la langue de la communauté de rattachement (le portugais), la langue majoritaire (l'allemand), les langues des autres communautés en Allemagne (voir à ce propos Melo-Pfeifer \& Schmidt, 2012a).

\section{Méthodologie}

Pour analyser les représentations des enfants par rapport aux langues de leurs répertoires, en général, et au Plco en particulier, nous avons adopté une méthodologie plus objectivante pour accéder aux représentations : le diagnostic de ces représentations étant notre objectif central, nous avons observé les 956 dessins et comptabilisé toutes les occurrences qui pointaient vers une langue (ou un peuple, une culture, ...). Les noms des 
langues n'ont pas été pris en compte parce qu'ils ne véhiculaient pas de représentations par rapport aux langues qu'ils nommaient, sauf quand les caractères utilisés font recours à des stéréotypes (par exemple, le "t" simulant la Tour Eiffel).

Les dessins recueillis configurent ce que l'on peut appeler des produits multimodaux (Mayers, 2009) plurilingues, puisqu'ils combinent l'écrit en plusieurs langues et l'image, généralement dans une relation de complémentarité. Parce que ces éléments mettent en évidence différents aspects, nous avons analysé les représentations visuelles et écrites en séparé. Comme l'illustre l'exemple suivant, une grande partie des dessins d'enfants sont multimodaux, ce qui implique l'analyse, en complémentarité ou en coordination, de mots et d'éléments picturaux :

Image 1. Les dessins d'enfants comme productions multimodales (Melo-Pfeifer \& Schmidt, 2012a).

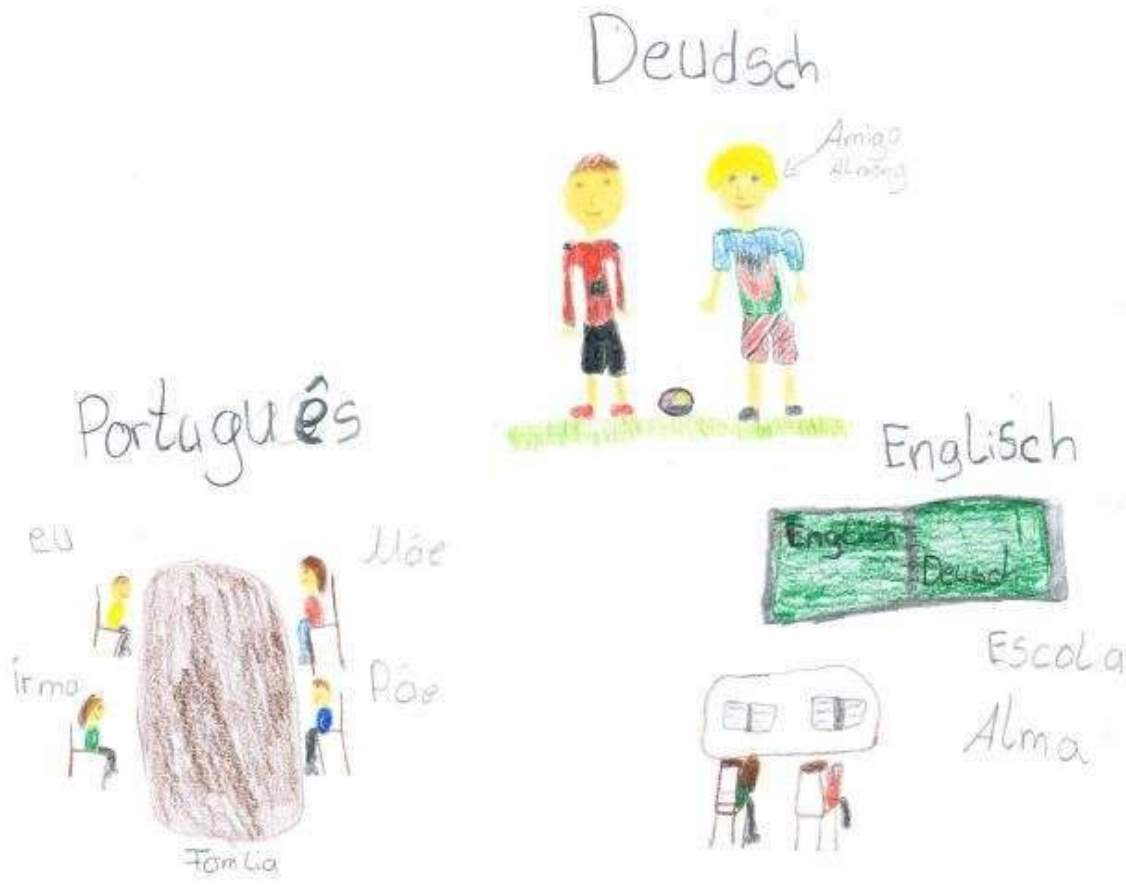

En outre, comme l'illustre cet exemple, il faut toujours dissocier les éléments attachés à chacune des langues représentées afin de cibler les résultats. Dans ce dessin, par exemple, l'amitié est associée à la langue allemande, la famille à la langue portugaise et l'apprentissage scolaire à la langue anglaise. Ceci dit, nous procédons par décomposition et recomposition ou, dans d'autres mots, de l'analyse du détail à l'articulation avec l'ensemble de la composition.

Nous avons employé les catégories suivantes (Melo-Pfeifer \& Pinto, 2009) pour grouper les éléments analysés, suite à l'approche plus objectivante que nous avons caractérisée avant :

- objet d'appropriation - concernant la facilité/difficulté d'apprentissage et d'usage ou l'éloignement/proximité par rapport à la langue nationale (l'allemand), d'autre(s) LM(s) ou à d'autres LE étudiées ;

- objet affectif - décrit le rapport affectif apprenant - langue - culture - peuple(s) ;

- objet de pouvoir - perception concernant la LCO en tant qu'atout en ce qui concerne le pouvoir économique et professionnel ; 
- objet culturel - liste les références culturelles à la langue et à la culture portugaises (notamment la gastronomie, les vêtements typiques, la littérature, la musique, l'architecture, la géographie ou l'histoire) ;

- instrument de construction de rapports interpersonnels et inter-groupaux - perceptions concernant le rôle de la langue dans la construction (ou limitation) des rapports entre citoyens et/ou peuples, entre les éléments de la même communauté linguistique (le "ingroup") ou entre des individus appartenant à différentes communautés (les "out-groups").

\section{Représentations concernant le portugais comme LCO} Allemagne (Melo-Pfeifer \& Schmidt, 2012a). Les enfants qui ont participé à notre étude ont des contacts réguliers avec cette communauté, notamment à travers la fréquentation des cours de Plco (qui ne sont ouverts que lorsqu'il y a au moins une quinzaine d'apprenants, tous niveaux et âges compris). Dans ce sens, ces enfants restent en contact avec un micro-cosmos sociolinguistique où la langue portugaise est l'une (ou même, dans certaines situations, la plus importante) des ressources linguistiques. Ainsi, notre public est au sein d'un contexte où circulent des images/représentations de la langue et culture portugaise : ces images circulent entre les membres de la communauté et sont encore mises en miroir, soit par les citoyens du pays d'accueil, soit par les autres communautés migrantes. visuelles) à la langue et à la culture portugaises :

Tableau 2. Représentations concernant le Plco.

\begin{tabular}{|l|l|l|l|l|}
\hline Catégories d'analyse & Exemples ${ }^{2}$ & $\begin{array}{l}\text { Références } \\
\text { écrites }\end{array}$ & $\begin{array}{l}\text { Références } \\
\text { dessinées }\end{array}$ & Total \\
\hline Objet d'apprentissage & École, maître, pronoms & 27 & 19 & 46 \\
\hline Objet affectif & Joli, ma famille & 126 & 91 & 217 \\
\hline Outil d'Empowerment & Je suis fort & 9 & - & 9 \\
\hline Objet culturel & Lisbonne, Ronaldo & 24 & 469 & 493 \\
\hline Outil de construction de & $\begin{array}{l}\text { Salut, Comment ça va?, je } \\
\text { parle le portugais à la } \\
\text { maison }\end{array}$ & 480 & - & 480 \\
\hline Autres / indéfinies & Livre, arbre, table interpersonnels & 45 & - & 45 \\
\hline & Total & 711 & $57+$ & 1280 \\
\hline
\end{tabular}

Ce tableau met en évidence le fait que, malgré le statut d'objet "para-scolaire" des cours de Portugais LCO, cette langue est surtout envisagée en tant qu'objet culturel, avec une forte valeur communicative, soit au sein de la famille, soit au sein de la communauté (image 5). Cette présence accrue de la culture portugaise dans notre corpus peut être 
considérée assez étonnante si l'on considère que la langue et la culture portugaises ne sont pas fréquentes dans le paysage linguistique et culturel allemand (malgré l'importante présence de la communauté portugaise en Allemagne. Comme nous l'avions affirmé dans un travail précédent, cette analyse met en évidence le fait que "the family (and the Portuguese community), as well as the PHL classes, are major vehicles for the transmission, the identification with and the recognition of Portuguese cultural aspects" (Melo-Pfeifer \& Schmidt, 2012a: 18).

41 Nous essaierons à présent de rendre plus explicites les choix des enfants en termes de représentations choisies, en illustrant nos propos à l'aide de la convocation, assez fréquente, des dessins recueillis. Pour mieux comprendre la portée de chaque catégorie de notre étude, nous présenterons des tableaux-synthèse avec les 5 éléments les plus présents par catégorie. Du fait du manque de représentativité de la catégorie "Outil d'Empowerment", nous ne pourrons pas en faire une analyse.

\section{Représentations culturelles concernant le Plco}

Le tableau 3 rend compte des associations les plus fréquentes, dans les dessins analysés, à la culture portugaise et le pourcentage par rapport à la totalité par catégorie (cf. tableau 1) :

Tableau 3. Les représentations culturelles les plus fréquentes.

\begin{tabular}{|l|l|l|l|}
\hline Éléments associés & Mode & Total de références & $\%$ \\
\hline Drapeau du Portugal & Dessin & 424 & $86 \%$ \\
\hline Football & Dessin et écrit & $20(18+2)$ & $4 \%$ \\
\hline Poisson & Dessin & $8(5+3)$ & $1,6 \%$ \\
\hline Église & Dessin et écrit & $5(4+1)$ & $1 \%$ \\
\hline Porto & Écrit & 4 & $0,8 \%$ \\
\hline
\end{tabular}

Ces résultats mettent en évidence une image assez stéréotypée de la culture portugaise et un imaginaire restreint et folklorique. Le dessin est, dans notre corpus, le mode privilégié de représentation des symboles attachés à la culture portugaise. En même temps, ces éléments culturels sont représentés soit au sein de la communauté portugaise en Allemagne (attachés au vécu en communauté minoritaire), soit au Portugal (attachés au vécu au sein de la communauté "de départ"); ils sont aussi présents à l'école (dite "parallèle", image 2) et en dehors de l'école. Ci-dessous, un dessin où les acquisitions scolaires (ici, en mathématique, quoique ce ne soit pas le but central des cours) vont de pair avec des références culturelles moins scolaires et plutôt ordinaires (les clubs de football "Porto" et "Benfica") : 


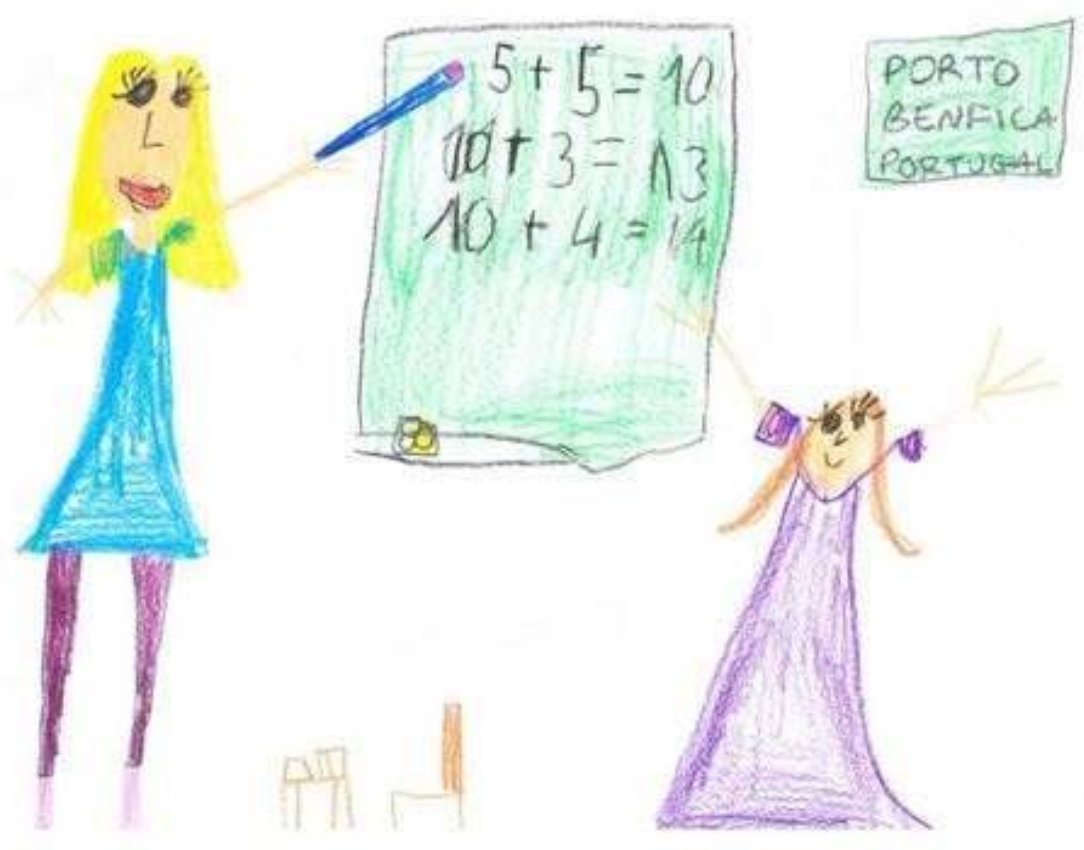

De son côté, l'image 3 fait une espèce de tour de table de l'imaginaire culturel lié à différentes langues : la "pizza", le "spaghetti" et la tour de Pise pour l'Italie, le "kebab" pour les Arabes, le "brezel" pour les Allemands et le "bacalhau" pour les Portugais.

Image 3. Représentations stéréotypées des langues-cultures.

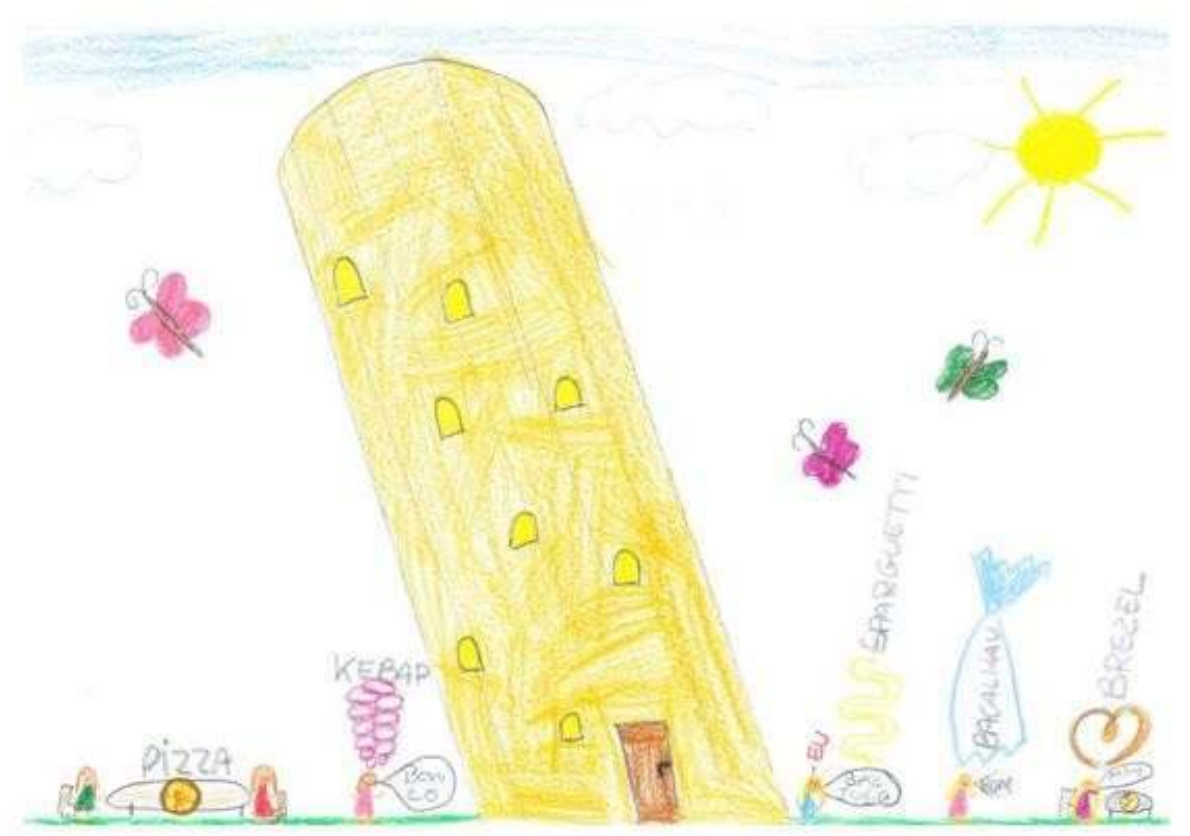

Ce dessin montre que, plus important que les images des langues d'origine ou de la langue du pays d'accueil, c'est l'environnement sociolinguistique qui semble attirer les enfants. Si Blommaert parlait de "bits of language" (2010) pour se référer aux ressources 
plurilingues mobiles des sujets, nous pourrions ici proposer, en effet, l'expression "bits of cultures" pour décrire ces ressources (inter)culturelles qui ont des sens précis dans des situations précises et qui donnent un sens aux vécus plurilingues et interculturels des enfants. Ceci dit, ces ressources, que ce soient les "bits of language" ou les "bits of culture", s'intègrent dans un imaginaire dynamique où les LCO et la langue du pays d'accueil ne sont que deux des ressources sémiotiques disponibles et mobilisées par les enfants.

Par contre, la représentation linéaire des différentes cultures dans ce dessin semble illustrer une société où les cultures co-existent mais ne se mélangent pas (des sociétés multilingues), désignée en allemand par le sympathique (mais à portée limitée) "multikulti".

\section{Représentations concernant le Plco en tant qu'outil de construction de rapports interpersonnels}

Notre analyse des dessins a mis en évidence une forte visée interpersonnelle dans le développement des répertoires linguistiques en Plco. Les expressions les plus présentes mettent en avant les besoins de communication des enfants dans des situations quotidiennes d'usage de cette langue :

Tableau 4. Les représentations socialisantes les plus fréquentes associées au Plco.

\begin{tabular}{|l|l|l|l|}
\hline Éléments associés & Mode & Total de références & $\%$ \\
\hline "Olá" / salut & Écrit & 287 & $60 \%$ \\
\hline " (eu) sou o/a" / Je suis & Écrit & 52 & $11 \%$ \\
\hline "(eu) chamo-me" / je m'appelle & Écrit & 35 & $7 \%$ \\
\hline "O meu nome é" / Mon nom, c'est & Écrit & 16 & $3,3 \%$ \\
\hline "Bom dia" / Bonjour & Écrit & 15 & $3 \%$ \\
\hline
\end{tabular}

Ceci dit, c'est le besoin d'entrer en contact, de connaître et de se faire connaître qui semblent pousser l'usage de la langue portugaise en contexte allemand. Nous avons affaire ici à un "cours d'initiation" à la sociabilité en Plco qui, avec les références visuelles, nous donne un aperçu plus complet des fonctions attachées à l'acquisition linguistique : 
Comme d'autres illustrations commentées dans nos travaux précédents (Melo-Pfeifer \& Schmidt, 2012a), ce dessin met en relief la dimension interpersonnelle de l'usage de la LCO dans la société d'accueil, même si la "norme" n'est pas (ou plus) la même que dans la "société d'origine". Dans ce cas, on remarque l'utilisation constante des pronoms personnels en portugais ("eu" / je ; "tu" / tu) que l'on omet fréquemment à l'oral et à l'écrit lorsque le verbe marque déjà le sujet ("acho" pour la première personne singulier et "gostas" pour la deuxième personne singulier). C'est l'hybridation des connaissances linguistiques, puisque toutes les autres langues rapportées comme "connues" par les enfants font usage des pronoms personnels : "je crois", "ich glaube", "I think".

La même observation peut se faire à propos de l'occurrence assez fréquente de l'expression "o meu nome é" (tableau 4), peu commune (même étrange) en portugais (en portugais du Brésil on dirait plutôt "Meu nome é "). Ce phénomène dénonce l'instabilité des "normes": l'allemande ("Meine name ist...") et la portugaise (le déterminant possessif est toujours précédé d'un déterminant, ici "meu" et "o", respectivement) - d'où la construction "O meu nome é...". Ceci dit, plus que la référence à la "norme", c'est l'usage situé des ressources disponibles, mélangées, hybrides, qui semble être au cœur des rapports interpersonnels, de leur construction et de leur maintien. On pourrait même faire l'hypothèse que ce serait l'usage de la norme qui semblerait étrange et maladroite pour ces enfants.

\section{Le Plco en tant qu'objet affectif}

51 Le Plco possède une forte dimension affective, comme nous l'avons déjà conclu à travers la comparaison avec les autres langues illustrées, langue du pays d'accueil et langues de 
l'école comprises (Melo-Pfeifer \& Schmidt, 2012a). Ici, images et paroles se combinent pour former une image idéalisée de la LCO :

Tableau 5. Le Plco comme objet affectif

\begin{tabular}{|l|l|l|l|}
\hline Éléments associés & Mode & Total de références & $\%$ \\
\hline Soleil & Dessin et écrit & $38(32+6)$ & $17,5 \%$ \\
\hline Fleurs & Dessin & 19 & $9 \%$ \\
\hline Plage & Dessin et écrit & $15(13+2)$ & $7 \%$ \\
\hline Père & Écrit et dessin & $10(9+1)$ & $4,6 \%$ \\
\hline Mère & Écrit et dessin & $10(9+1)$ & $4,6 \%$ \\
\hline
\end{tabular}

52 Si le soleil et la plage ont été considérés comme des références affectives plutôt que comme des références culturelles, c'est que leur usage se fait toujours dans des situations soit de comparaison avec le vécu en Allemagne soit de détente au Portugal. C'est donc plutôt l'usage de la référence que sa valeur culturelle "inhérente" (ici "climat" et "géographie") qui ont guidé leur classification. Ceci dit, c'est l'association de la LCP au beau temps, aux vacances et aux moments de détente qui semble embellir le rapport des enfants à cette langue.

Comme le tableau 5 le rapporte et le dessin 5 l'illustre, le rapport affectif au Plco est médié par la mention, souvent présente, à la famille (voir encore image 1) : 
Image 5. Le rapport enfant-mère en Plco ("Salut, maman, je t'aime / Moi aussi je t'aime, parce que tu es ma fille").

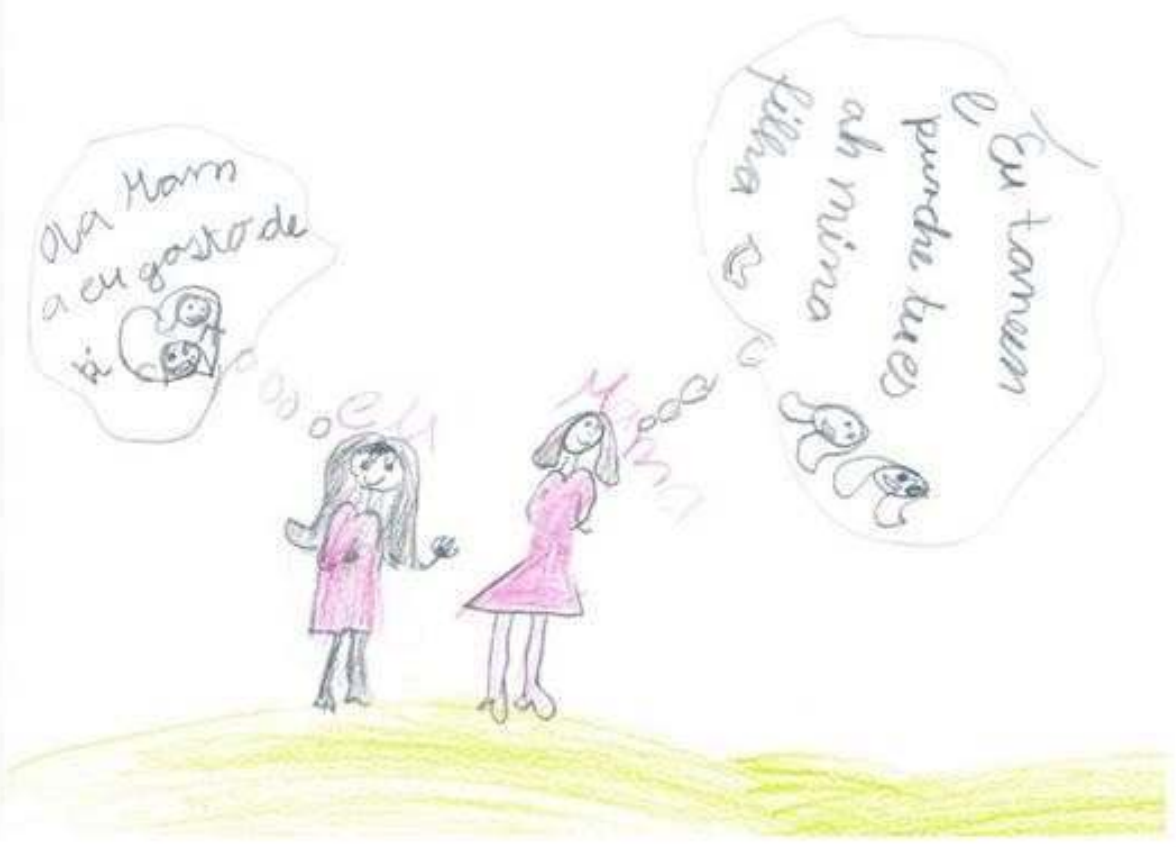

On voit bien ici comment l'image du rapport fille-mère passe par l'utilisation du Plco que l'on devine utilisé en contexte familial. Ici, la langue d'origine acquiert une fonction assez spécifique : la langue de communication à la maison, où l'on crée des liens familiaux. Dans ce dessin, c'est l'enfant qui prend l'initiative de la parole et donc tout le risque inhérent à la communication dans une langue donnée, ce qui semble déjà dépasser l'état de timidité linguistique (Krashen, 1998) qui caractérise beaucoup de locuteurs d'une langue d'origine (même si, dans ce cas, nous avons affaire à un climat affectif assez positif et protecteur).

L'image 6 représente un enfant qui se voit comme plurilingue portugais, allemand et anglais. Les langues sont représentées verticalement, dans l'ordre présentée avant, et la même information est répétée en colonne (à gauche) : " salut, je m’appelle Bruno". Ici, nous pourrions faire l'hypothèse que c'est la valeur communicative des langues (LCO, L Nationale et LE) qui est protagoniste. Cependant, ce dessin reflète deux niveaux de représentation de la LCO: à droite, l'enfant utilise le portugais pour donner des informations personnelles concernant son état d'esprit ("Salut, je suis heureux"). 
Image 6. Le Plco comme langues des émotions ("Salut, je m'appelle Bruno (PT, DE, EN). / Salut, je suis heureux (PT)").
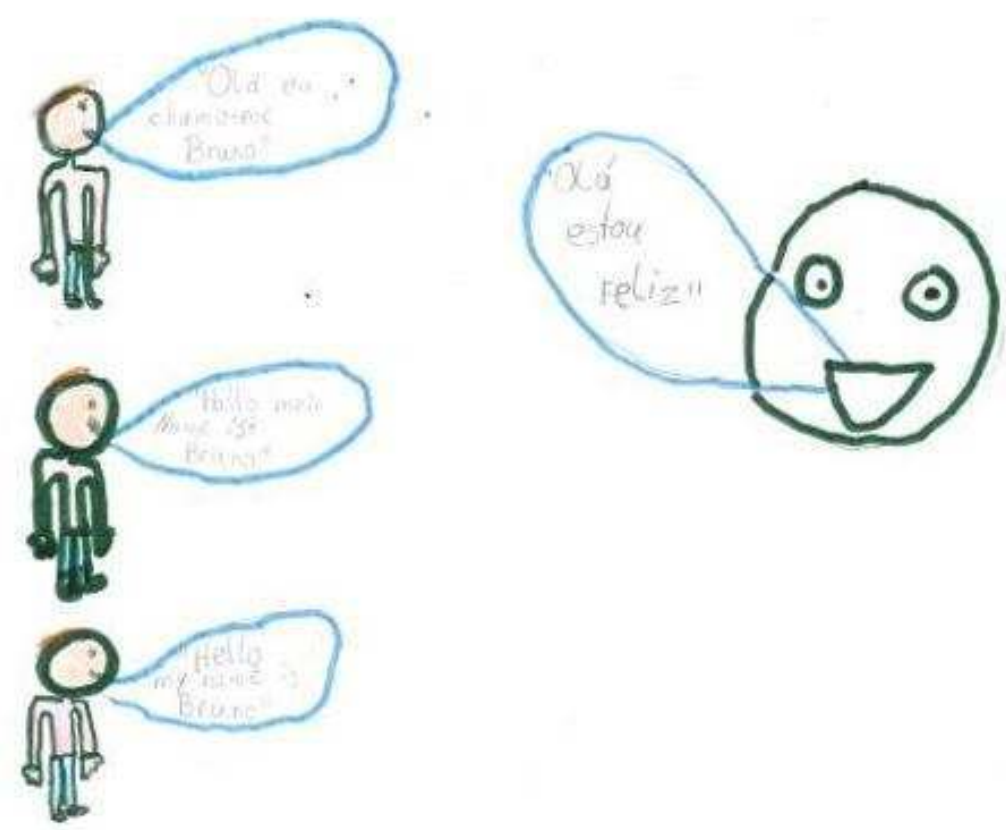

Il faut remarquer que, si dans les représentations dans la colonne, l'enfant à esquissé tout son corps dans une même position et direction, ce visage heureux et nettement plus grand regarde le public, comme s'il voulait le rendre auteur et personnage de ce "comic". Il y a là donc un essai de construction d'un rapport d'intimité, par l'image et par la langue, avec les destinataires de ce dessin (le professeur? la classe ?), eux aussi sujets parlant de Plco.

\section{Représentations scolaires du Plco}

57 L'imaginaire de notre public concernant le portugais en tant qu'objet d'acquisitionapprentissage met en scène des représentations matérielles de l'espace scolaire. Ainsi, peut-être à cause des conditions de production de ces dessins, les enfants semblent envisager surtout l'école comme environnement d'apprentissage de la langue d'origine. Des références à des situations d'acquisition en famille, à travers la communication au sein de la communauté ou d'autres moyens sont absentes. Même si ces représentations ne sont pas fréquentes, nous confirmons une grande valeur attribuée aux cours de LCO dans l'imaginaire de ce que signifie apprendre à maitriser cette langue.

Tableau 6. Le PIco comme objet scolaire.

\begin{tabular}{|l|l|l|l|}
\hline Éléments associés & Mode & Total de références & $\%$ \\
\hline École & Dessin & 14 & $30 \%$ \\
\hline Professeur & Dessin et écrit & $9(3+6)$ & $19,5 \%$ \\
\hline Je suis à l'école maternelle & Écrit & 3 & $6,4 \%$ \\
\hline
\end{tabular}




\begin{tabular}{|l|l|l|l|}
\hline Cours & Écrit & 2 & $4 \%$ \\
\hline
\end{tabular}

Dans ce sens, ces résultats vont de pair avec d'autres études qui pointent une vision assez scolarisée de l'apprentissage des langues et une survalorisation du rôle de l'école, du professeur et des manuels de langue (Araújo e Sá \& Pinto, 2006), comme on le voit dans l'image 7 :

Image 7. Le cours de Plco: une classe comme les autres.

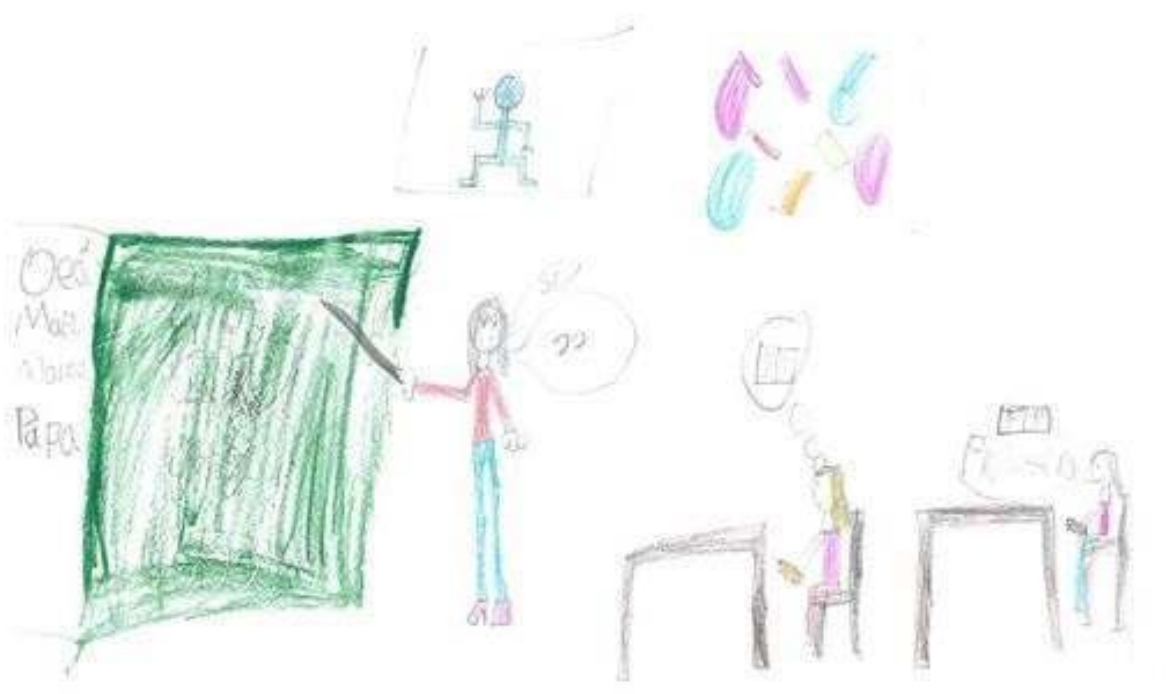
dans l'acquisition des langues ou dans l'acquisition d'autres matières scolaires (d'une façon très peu attentive à la diversité des répertoires des enfants), notre public représente souvent une salle de classe qui mobilise plusieurs ressources linguistiques (la LCO et l'allemand et encore fréquemment leur première LE, l'anglais) et qui les compare d'un point de vue lexical. Il semble souvent que l'on fait l'effort d'établir un rapport entre toutes les ressources linguistiques des enfants, le développement d'une langue ayant un impact sur le développement des autres (il $\mathrm{y}$ a des dessins où l'on peut deviner la rentabilisation des ressources allemandes dans l'acquisition de la langue d'origine). Nous pourrions donc affirmer que la comparaison interlinguistique, telle que prônée par des approches plurielles telles que l'intercompréhension (en dehors des familles de langues) ou l'éveil aux langues, commence à combattre l'"habitus monolingue" du moins dans les cours de langue.

Même si la survalorisation du rôle des cours est souvent reconnue, comme nous l'avions affirmé auparavant, le fait que ces cours aient leur place hors horaire scolaire entraine des moments de démotivation, car où l'on doit faire un effort supplémentaire pour y participer à la fin de la journée (image 8) : 


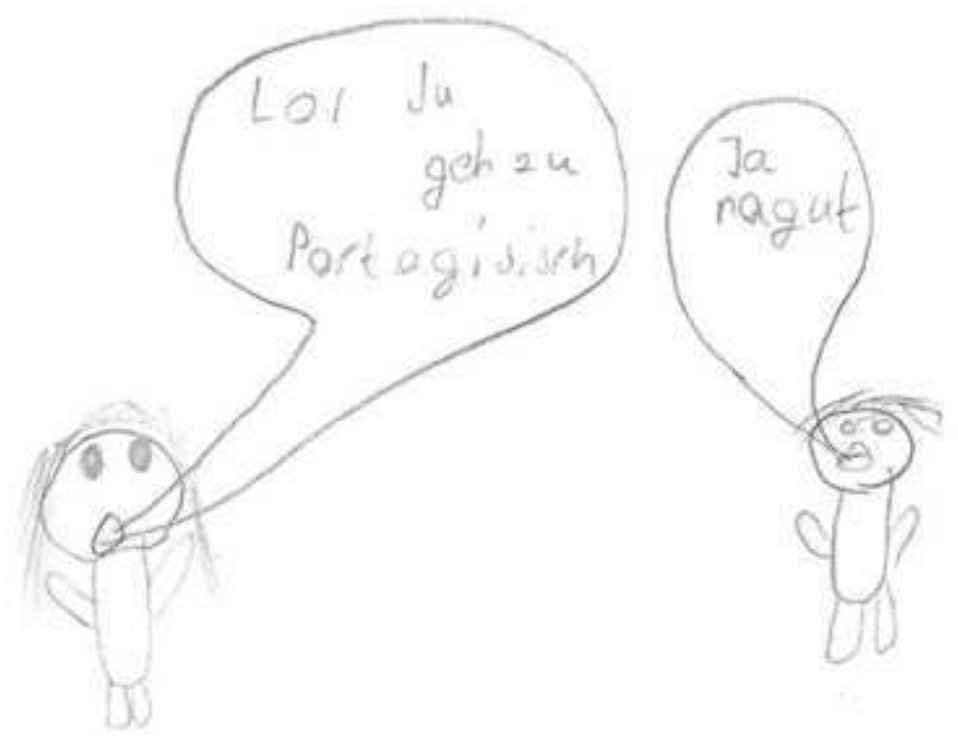

61 Ce dessin est un peu ambigu en termes d'acteurs : est-ce la mère qui "oblige" l'enfant à fréquenter les cours de Plco ? Est-ce un camarade qui essaye de motiver son copain? Quoi qu'il en soit, la réponse est conformiste et très peu de convaincante (confirmée par le gris du dessin).

\section{"Profil d'apprenant" et perspectives de développement du Plco: esquisse d'un rapport avec les approches plurielles}

Nos résultats mettent en évidence l'hétérogénéité des répertoires plurilingues des enfants, d'un côté, et des répertoires en Plco de l'autre côté. Parmi d'autres différences, c'est aussi le rapport à la langue portugaise qui est différent. Il faut encore souligner que ce n'est pas tant la "norme" portugaise (ou des autres langues) qui est présente dans les éléments écrits par les enfants, mais les représentations des ressources multilingues, c'est-à-dire, leur valeur pragmatique et socioaffective-identitaire. Dans cette perspective, nous faisons l'hypothèse que ce n'est pas le contenu linguistique et verbal qui est le plus important dans ces dessins, mais la mise en scène, par les enfants, de leurs ressources individuelles et de leurs identités multilingues (qui ne peuvent pas se circonscrire au Plco et à l'allemand Langue Nationale).

Or, d'après notre expérience et observations de terrain, les trajectoires d'enseignement semblent ne pas savoir se confronter à cette diversité de rapports et de ressources et les formations des enseignants mises en œuvre pour répondre à cette hétérogénéité semblent faire défaut un peu partout. Tout se passe comme si une simple importation de modèles théoriques et pragmatiques et de formation de professeurs pourrait suffire à répondre aux besoins et aux demandes de ce public apprenant, auquel on attache les labels LM ou LE pour "mieux" cadrer les approches d'enseignement et les méthodologies 
(ici assimilées au "manuel de langue"). Et ainsi, pour mieux transmettre une "norme" linguistique, voire culturelle.

D'un autre côté, les résultats de cette étude sont encore très parlants si l'on rappelle celle de Melo-Pfeifer \& Pinto (2009), auprès d'étudiants universitaires par rapport au Français Langue Étrangère, où les catégories "Objet culturel" et "Outil de construction de rapports interpersonnels", ici les plus représentatives, étaient les moins visibles. Nous faisons l'hypothèse que le contenu des représentations par rapport aux langues change en fonction de l'âge des apprenants et du statut de la langue visée (ici LCO; LE dans l'étude de Melo-Pfeifer \& Pinto). Cette hypothèse est cimentée par le fait que les représentations de pouvoir et d'utilité associées à la langue sont beaucoup plus présentes auprès des étudiants par rapport à l'apprentissage d'une LE, considérée comme un investissement professionnel. Les enfants sont encore en train de développer un rapport affectif à la langue, à une langue à statut sociolinguistique, socioculturel et socioaffectif particulier. Par conséquent, l'argument professionnel et instrumental de l'apprentissage du Plco fait très peu de chemin auprès des enfants, langue à laquelle ils attachent plutôt une grande valeur affective.

Dans ce cadre, et en rapport avec les approches plurielles pour les langues et les cultures (Candelier et al, 2007), nous croyons qu'une intégration réussie de ces enfants et la valorisation de leurs ressources (linguistique et culturelles) plurielles pourraient passer par leur rentabilisation en classe, pas seulement de LCO, du fait même que les approches plurielles reconnaissent les atouts des différentes langues et prennent les similitudes et les différences entre les langues comme des ressources à employer de façon consciente et rentable (et non pas comme une adversité à surmonter).

Ainsi, les LCO, comme conseillé par un certain nombre de chercheurs, devraient se mettre en place en tant que ressource, sans être la proie des discours sur l'inégalité sociale ou l'échec scolaire. Le développement de stratégies de comparaison interlinguistiques (telles que prônées par les approches intercompréhensives), la mobilisation, en tant qu' "outils" d'apprentissage sociolinguistique, de membres des communautés migratoires dans la classe et à l'école (un peu dans la ligne de l'éveil aux langues et de l'approche interculturelle) et l'apprentissage des matières scolaires par les langues et par le contact avec des ressources informatives en plusieurs langues (dans la ligne de la didactique intégrée), sont des stratégies possibles à mettre en place dans des "complex transnational spaces" (Byrd Clark, 2012) - notamment éducatifs -, où les normes, tout comme les identités, sont éphémères, co-construites, co-négociées et multiples.

Par exemple, en tant que langue dans laquelle les sujets possèdent une compétence partielle (la compréhension étant souvent bien plus développée que la production), la langue d'origine rend compte des potentiels des approches intercompréhensives: on mobilise le déjà-là, souvent méconnu, souvent désinvesti (parce qu'acquis en dehors de l'école ou parce que connoté avec une minorité), pour bâtir un espace de rencontre de langues, où l'accès au sens, par le biais d'une compétence de compréhension accrue, est l'un des ingrédients de la co-construction du sens en situation de communication. Pour ce qui est de l'Approche Interculturelle, la connaissance d'une langue-culture en plus apporte des atouts complémentaires pour comparer, confronter et rapprocher les systèmes de valeurs, les croyances, les stéréotypes, les références culturelles plus ou moins transversales, les éléments plus ou moins originaux de chaque culture. Pour ce qui est, finalement, de l'Éveil aux Langues, la langue d'origine permet un ancrage cognitif et 
affectif supplémentaire, une ressource individuelle d'accès au sens et un potentiel de partage dans les tâches collaboratives de découverte des langues.

\section{BIBLIOGRAPHIE}

Araújo e Sá, Mํa H. et al, (2012). "Representations of languages within educational settings". Symposium au Colloque Pluri-L, Vers le Plurilinguisme? 20 ans après. 21-23 mars 2012, Universités de Nantes-Angers-Le Mans.

Araújo e Sá, Mª. H. \& Pinto, S. (2006). "Imagens dos outros e suas línguas em comunidades escolares: produtividade de uma temática de investigação em educação linguística". In R. Bizarro (org.), A Escola e a diversidade cultural- multiculturalismo, interculturalismo e educação. Porto: Areal Editores. pp 227-240.

Araújo e Sá, Mª H. \& Schmidt, A. (2008). "The awareness of language prestige: the representations of a portuguese school community on important languages". In M. Candelier, G. Ioannitou, D. Omer \& M.-T. Vasseur (Dirs.), Conscience du plurilinguisme: pratiques, représentations et interventions. Rennes, France: Presses Universitaires de Rennes. pp. 109-124.

Bertucci, M.-M. \& Corblin, C. (org.) (2007). "Enseigner les langues d'origine", Le Français aujourd'hui, 158. URL http://www.cairn.info/revue-le-francais-aujourd-hui-2007-3.htm.

Blommaert, J. (2010). The Sociolinguistics of Globalization. Cambridge : Cambridge University Press. Bono, M. \& Melo-Pfeifer, S. (2012). "La compétence plurilingue dans une perspective socioconstructiviste et (co-)actionnelle”. In G. Alao, M. Derivry, S. Yun-Roger \& E. Suzuki (2012), Didactique plurilingue et pluriculturelle : l'acteur en contexte mondialisé. Paris : Éditions des Archives Contemporaines. pp. 61-72.

Boyer, H. (éd.) (1997). Plurilinguisme : "contact" ou "conflit" de langues?. Paris : L'Harmattan. Brinton, D., Kagan, O. \& Baukus, S. (ed.) (2007). Heritage Language Education: a new field emerging. New York : Routledge.

Byrd Clark, J. (ed.) (2012). Journeys of Integration between multiple worlds: reconceptualising multilingualism through complex transnational spaces. International Journal of Multilingualism (Special Issue), $9 / 2$.

Candelier, M. (1997). "Catégoriser les représentations". In G. Zarate (coord.), Les Représentations en Didactique des Langues et Cultures. Notions en Question, 2. Paris: Crédif - Didier. pp. 43-65.

Candelier, M. et al (ed.) (2007). Cadre de référence pour les approches plurielles des cadres et des cultures. Graz : European Centre for Modern Languages.

Candelier, M. \& Hermann-Brennecke (1993). Entre le choix et l'abandon. Les langues étrangères à l'école vues d'Allemagne et de France. Paris : Didier.

Carreira, M. \& Kagan, O. (2011). "The results of the National Heritage Language Survey: implications for teaching, curriculum design, and professional development". Foreign Language Annals, vol. 44, n¹, pp. 40-64. 
Castellotti, V. (2010). "Les enseignements de "langues (et cultures) d'origine". Chronique d'une disparition opportune ?". In L. Cadet, J. Goes \& J.-M. Mangiante (dir.), Langue et intégration. Bruxelles : Peter Lang. pp. 83-94.

Castellotti, D. \& Moore, D. (2010). Valoriser, mobiliser et développer les répertoires plurilingues pour une meilleure intégration scolaire. Strasbourg : Conseil de l'Europe. URL http://www.coe.int/t/dg4/ linguistic/Source/Source2010_ForumGeneva/4-ValoriserCastellottiMoore_FR.pdf

Castellotti, V. \& Moore, D. (2002). Social Representations of Languages and Teaching. Reference Study, Language Policy Division, Council of Europe, Strasbourg. URL http://www.coe.int/T/E/ Cultural_Cooperation/education/Languages/Language_Policy/Policy_development_activities/ Studies/CastellottiMooreEN.pdf.

Cook, V. (2003) (ed.). Effects of the Second Language on the First. Clevedon : Multilingual Matters.

Coste, D., Moore, D. \& Zarate, G. (2009). Compétence Plurilingue et Pluriculturelle. Vers un Cadre Européen Commun de référence pour l'enseignement et l'apprentissage des langues vivantes : études préparatoires. Version révisée. Strasbourg : Conseil de l'Europe. URL http://www.coe.int/t/dg4/ linguistic/Source/SourcePublications/CompetencePlurilingue09web_fr.pdf.

Cuq, J.-P. (2003). Dictionnaire de Didactique du Français. Paris : Clé International.

Dabène, L. (2000). "Pour une didactique plurielle: quelques éléments de réflexion". In La didactique des langues dans l'espace francophone: unité et diversité. Actes du 6ème Colloque International de Acedle. pp. 9-13.

Dabène, L. (dir.) (1989). "Les langues d'origine des populations migrantes : un défi pour l'école française". Lidil, 2, Grenoble, Presses Universitaires de Grenoble.

De Pietro, J.-F. (1995). "Synthèse des résultats". In Commission française pour l'UNESCO (ed.), Stéréotypes culturels et apprentissage des langues. Programme d'études en matière d'éducation des Commissions européennes pour l'Unesco. pp. 309-321.

De Pietro, J.-F. \& Müller, N. (1997), "La construction de l'image de l'autre dans l'interaction. Des Coulisses de l'implicite à la mise en scène". In Bulletin suisse de linguistique appliquée, 65. pp. 25-46.

Deprez, C. (1997). "L'apprenant et ses langues: représentations métalinguistiques dans les entretiens autobiographiques". In G. Zarate (coord.), Les Représentations en Didactique des Langues et Cultures, Notions en Question, 2. Paris : Crédif - Didier. pp.77-101.

Fürstenau, S. \& Gomolla, M. (coord.) (2011). Migration und schulischer Wandel: Mehrsprachigkeit. VS Verlag.

Gajo, L. (2000). "Disponibilité sociale des représentations : approche linguistique". In B. Py (éd.), Analyse conversationnelle et représentations sociales. Unité et diversité de l'image du bilinguisme. Tranel, 32. pp. 39-53.

García, O. (2009). "Education, multilingualism and translanguaging in the $21^{\text {st }}$ century". In T. Skutnabb-Kangas, R. Phillipson, A. K. Mohanty \& M. Panda (ed.), Social Justice through Multilingual Education. Bristol: Multilingual Matters. pp. 140-158.

Gogolin, I. (1994). Der monolinguale Habitus der multilingualen Schule. München, New York : Waxmann.

Krashen, S. (1998). "Heritage Language Development: Some practical Arguments". In S. Krashen \& L. Tse \& J. McQuillan (ed.), Heritage Language Development. California: Language Education Associates. pp. 3-13. 
Krumm, H.-J. (2002). "'Französisch in den Beinen... um in die Ferne zu gehen" Sprachenporträts: ein Zugang zu Sprachbiographien". In Bulletin VALS-ASLA, 76. pp.197-202).

Little, D. (2010). Intégration linguistique et éducative des enfants et adolescents issus de l'immigration. Strasbourg : Conseil de l'Europe. URL http://www.coe.int/t/dg4/linguistic/Source/ Source2010_ForumGeneva/MigrantChildrenConceptPaper_FR.pdf.

Mavers, D. (2009). "Image in the multimodal ensemble : Children's drawing". In C. Jewitt (ed.), The Routledge Handbook of Multimodal Analysis. Oxon: Routledge. pp. 263-271.

Melo, S. (2006). Emergência e Negociação de Imagens das línguas em Encontros Interculturais Plurilingues em Chat. Thèse de doctorat. Aveiro : Universidade de Aveiro. URL http://biblioteca.sinbad.ua.pt/ Teses/2007001006.

Melo-Pfeifer, S. \& Pinto, S. (2009). "Évolution des images du FLE à l'université : une étude de cas au Portugal". In Les Cahiers de l'ACEDLE, 6. pp. 153-171. URL http://acedle.org/IMG/pdf/ Melo_Pinto_Cahiers-Acedle_6-1.pdf .

Melo-Pfeifer, S. \& Schmidt, A. (2012a). "Linking “Heritage Language" Education and Plurilingual Repertoires development: evidences from drawings of Portuguese pupils in Germany". In L1Educational Studies in Language and Literature, 12 (1-30). URL http://11.publication-archive.com/ public?fn=document\&id=2497\&repository=1 (co-édité par M. Byram, M. Fleming \& I. Pieper).

Melo-Pfeifer, S. \& Schmidt, A. (2012b). "Représentations croisées de la communauté portugaise, des enseignants et des apprenants de Portugais Langue d'Origine. Enjeux et perspectives didactiques". Intervention orale au Colloque International Diltec : "Langages - Cultures - Sociétés : interrogations didactiques", 20, 21 et 22 juin 2012, Université Paris 3 - Sorbonne Nouvelle, Paris (France).

Molinié, M. (ed.) (2009). Le dessin réflexif. Élément pour une herméneutique du sujet plurilingue. CergyPontoise : Université de Cergy-Pontoise.

Moore, D. \& Castellotti, V. (2011). "Dessins d'enfants, recherche qualitative, interprétation. Des poly-textes pour l'étude des imaginaires du plurilinguisme". In Ph. Blanchet \& P. Chardenet (dir), Guide pour la recherché en didactique des langues. Approches contextualisées. Paris : Éditions des Archives Contemporaines. pp. 118-132.

Moussouri, E. (2010). "Pratiques didactiques et représentations : un outil pour la conception d'une formation destinée aux enseignants des langues secondes/d'origine". In Les Cahiers de l'ACEDLE, 7/2 (139-168). URL http://acedle.org/IMG/pdf/Moussouri_Cahiers-Acedle_7-2.pdf.

Moscovici S. (1989). "Des représentations collectives aux représentations sociales: éléments pour une histoire". In D. Jodelet (dir.), Les représentations sociales. Paris: P.U.F. (79-103).

Perregaux, Ch. (2011). "Draw me a language! Understanding the Imaginary of Young Children". Child Health and Education, vol. 3, n¹. pp. 16-30.

Pinto, S. (2005). Imagens das línguas estrangeiras de alunos universitários portugueses. Dissertation de Master. Aveiro: Universidade de Aveiro.

Py, B. (2004). "Pour une approche linguistique des représentations sociales". In J.-C. Beacco (org.), Représentations métalinguistiques ordinaires et discours, Langages, 154. pp. 6-19.

Schmidt, A. (2011). Imagens das Línguas e Afectividade em contexto escolar. Um estudo sobre o Alemão. Thèse de doctorat. Aveiro : Universidade de Aveiro.

Zarate, G. (1993). Représentations de l'étranger et didactique des langues. Paris : Didier. 
Zarate, G. (1995). "La problématique de la relation à l'altérité : l'apprenant et l'enseignant de langue comme acteurs sociaux d'entre-deux. La politique linguistique et culturelle de l'Europe". In C. Briane \& A. Cain (org.), Quelles perspectives pour la recherche en didactique des langues? Paris : Institut National de Recherche Pédagogique. pp. 11-16.

Zarate, G. (1997). "La notion de représentation et ses déclinaisons". In G. Zarate (coord.), Les Représentations en Didactique des Langues et Cultures, Notions en Question, 2. Paris : Crédif - Didier. pp. 5-9.

Yanaprasart, P. (2002). "La dynamique des représentations dans les contacts interculturels". In P. Yanaprasart (ed.), Communiquer en milieu interculturel. Travaux neuchâtelois de linguistique, 36. pp. 59-79.

\section{NOTES}

1. En Allemagne, plusieurs états fédéraux embauchent les professeurs de LCO, étant responsables par leur formation et paiement.

2. Traduits de l'allemand et/ou du portugais.

\section{RÉSUMÉS}

Après une discussion critique du concept de Langue-Culture d'Origine et son rapport avec d'autres concepts désignant le rapport des sujets aux langues, nous présentons le projet "Images (de l'enseignement) du Portugais à l'Étranger", qui vise à rendre compte des images de la langue portugaise (qu'elle soit considérée LM, LE ou LCO) dans différents contextes. Dans cette étude, nous présenterons et discuterons les images recueillies auprès d'un public d'enfants "lusodescendants" en Allemagne à travers le recueil de leurs dessins, en classe de Langue Culture d'Origine, sous l'instruction "dessine-toi en train de parler les langues que tu connais". Les résultats permettent de cerner et de discuter le profil linguistique et socio-affectif de ce public et de présenter des implications pour une Didactique des LCO en rapport avec les approches plurielles des langues-cultures.

After critically discussing the concept of Heritage Language and considering the relationship between that concept and others designating the relationship individuals maintain with their languages, we will present the research project "Images of Portuguese (language, teaching and learning)". This project aims at detecting and analysing images of Portuguese and the practices used in its teaching and learning worldwide (whether it is a Mother Tongue, a Foreign Language or a Heritage Language), in different contexts. In this study, we will present and discuss the social representations "luso-descendent speaker" children living in Germany possess. These social representations were collected through drawings in the Portuguese Heritage Language classroom, under the instruction "draw yourself while speaking the languages you know". The results allow us to determine and to discuss the linguistic and socio-affective profiles of these children as well as to present some implications in terms of Heritage Language Education when related to multilingual approaches to languages and cultures. 
INDEX

Mots-clés : approches plurielles, Portugais Langue et Culture d'Origine, répertoires plurilingues, dessin réflexif

Keywords : multilingual approaches, Portuguese as Heritage Language, multilingual resources, reflective drawing

\section{AUTEURS}

\section{SÍLVIA MELO-PFEIFER}

Sílvia Melo-Pfeifer est docteure en Didactique des Langues et chercheure auprès du Centro de Investigação Didáctica e Tecnologia na Formação de Formadores (Cidtff) de l'Université d'Aveiro. Elle coordonne l'enseignement du portugais en Allemagne, auprès de l'Ambassade de Portugal à Berlin, et le projet "Images (de l'enseignement) du Portugais à l'Étranger", avec le soutien de Camões, ICL.

Courriel : smelo@ua.pt; silvia.pfeifer@camoes.mne.pt Adresse : Coordenação do Ensino Português na Alemanha, Embaixada de Portugal em Berlim, Zimmerstrasse 56, 10117 Berlin.

\section{ALEXANDRA SCHMIDT}

Alexandra SCHMIDT est docteure en Didactique de Langues et chercheure auprès du Laboratório Aberto para a Aprendizagem de Línguas Estrangeiras (Lale) de l'Université d'Aveiro.

Courriel : aschmidt@ua.pt; alexandra.schmidt@camoes.mne.pt Adresse : Coordenação do Ensino Português na Alemanha, Embaixada de Portugal em Berlim, Zimmerstrasse 56, 10117 Berlin. 